\title{
Cell-Engineered Human Elastic Chondrocytes Regenerate Natural Scaffold In Vitro and Neocartilage with Neoperichondrium in the Human Body Post-Transplantation
}

\author{
Hiroko Yanaga, M.D., Ph.D., Keisuke Imai, M.D., Ph.D., ${ }^{1}$ Mika Koga, M.Sc., and Katsu Yanaga, M.D. ${ }^{1}$
}

We have developed a unique method that allows us to culture large volumes of chondrocyte expansion from a small piece of human elastic cartilage. The characteristic features of our culturing method are that fibroblast growth factor-2 (FGF2), which promotes proliferation of elastic chondrocytes, is added to a culture medium, and that cell-engineering techniques are adopted in the multilayered culture system that we have developed. ${ }^{1-4} \mathrm{We}$ have subsequently discovered that once multilayered chondrocytes are transplanted into a human body, differentiation induction that makes use of surrounding tissue occurs in situ, and a large cartilage block is obtained through cartinogenesis and matrix formation. We have named this method two-stage transplantation. We have clinically applied this transplantation method to the congenital ear defect, microtia, and reported successful ear reconstruction. ${ }^{4}$ In our present study, we demonstrated that when FGF2 was added to elastic chondrocytes, the cell count increased and the level of hyaluronic acid, which is a major extracellular matrix (ECM) component, increased. We also demonstrated that these biochemical changes are reflected in the morphology, with the elastic chondrocytes themselves producing a matrix and fibers in vitro to form a natural scaffold. We then demonstrated that inside the natural scaffold thus formed, the cells overlap, connect intercellularly to each other, and reconstruct a cartilage-like three-dimensional structure in vitro. We further demonstrated by immunohistochemical analysis and electron microscopic analysis that when the multilayered chondrocytes are subsequently transplanted into a living body (abdominal subcutaneous region) in the two-stage transplantation process, neocartilage and neoperichondrium of elastic cartilage origin are regenerated 6 months after transplantation. Further, evaluation by dynamic mechanical analysis showed the regenerated neocartilage to have the same viscoelasticity as normal auricular cartilage. Using our multilayered culture system supplemented with FGF2, elastic chondrocytes produce an ECM and also exhibit an intercellular network; therefore, they are able to maintain tissue integrity post-transplantation. These findings realized a clinical application for generative cartilage surgery.

\section{Introduction}

$\mathbf{R}$ ECONSTRUCTION OF HARD-TISSUE defects associated with a craniofacial lesion, including injuries and abnormalities, is a major challenge in plastic surgery. For reconstructive surgery, a large volume of cartilage is necessary for reconstruction and remodeling. However, autologous cartilage grafts can be harvested in only small and limited volumes, and are associated with donor-site morbidity. Transplantation of cultured autologous chondrocytes has some advantages, such as minimal burden and morbidity at the donor site, and a large volume of chondrocytes is obtainable.

Many researchers have attempted transplantation of cultured human chondrocytes in animal models, ${ }^{5-13}$ but have been unable to clinically apply these. This is because cultured chondrocytes grow in a monolayer fashion in vitro, separate from each other, ${ }^{14,15}$ and do not maintain the integrity of cartilage tissue after transplantation. As a consequence, these chondrocytes are absorbed after transplantation. It has been reported that with a chick limb bud, murine cell line, human articular chondrocytes, etc., when hyaline chondrocytes are dropped onto a Petri dish at a high density in microunits of $10-20 \mu \mathrm{L}$, that is, in a micromass spot culture or micromass pellet culture, the phenotype of the chondrocytes is maintained, and a more tissue-like environment is created. ${ }^{16-18}$ However, if the chondrocytes are seeded or inoculated, at a high density from the beginning, the cells form a mass and do not undergo expansion growth.

\footnotetext{
${ }^{1}$ Yanaga Clinic and Tissue Culture Laboratory, Chuo-ku, Fukuoka, Japan.

${ }^{2}$ Department of Plastic Surgery, Osaka City General Hospital, Miyakojima-ku, Osaka, Japan.
} 
We believe that a large amount of elastic chondrocytes is necessary for the use of clinical facial reconstruction. We thus developed a multilayered culture system in which, first, fibroblast growth factor-2 (FGF2), ${ }^{19-24}$ known as a proliferation promoter of chondrocytes, is added to a medium to make cells multiply and undergo sufficient expansion, and these cells are then layered on top of each other and seeded. ${ }^{1-4}$ With this system, a large volume of chondrocytes was obtained, since the cells are prevented from becoming dispersed and are connected mutually by being multilayered. We also considered that if the chondrocytes can be arranged multilayered to form a three-dimensional (3D) structure, the intercellular connections will be strong and cells will not be absorbed readily after transplantation.

We then proposed two transplantation methods for the clinical application of multilayered chondrocytes. We first developed (1) a one-stage injection-transplantation method. In this method, the multilayered chondrocytes are injectiontransplanted to the craniofacial region directly. We have reported a successful clinical application of this method for tissue defects, such as injury, anomaly, and deformity cases of the craniofacial region. ${ }^{1-3}$ We then developed (2) a twostage transplantation method. In the two-stage transplantation process, the multilayered chondrocytes formed a neocartilage with neoperichondrium in the abdominal wall of a patient over a 6-month period; this method has been successfully clinically applied to ear reconstruction in microtia, as we previously reported. ${ }^{4}$ We have already used elastic chondrocyte transplantation to treat 110 patients with craniofacial lesions safely for 10 years.

The purpose of our study was to demonstrate that when using the multilayered culture system supplemented with FGF that we have developed the chondrocytes form multilayers, differentiation is induced and they produce and secrete hyaluronic acid (HA) and then form a natural scaffold, all in vitro. The HA binds with aggrecan, a cartilaginous proteoglycan that is a principal component of the main extracellular matrix (ECM) of cartilage and forms a natural scaffold in vitro. We thus measured HA in the medium to which FGF was added. We also performed an electron microscopic analysis to morphologically observe that dedifferentiated chondrocytes redifferentiate in the multilayered culture system.

We then demonstrated by immunohistochemical analysis that when in the application of the two-stage transplantation process for the clinical example of microtia, the multilayered chondrocytes are transplanted into the abdominal subcutaneous region, and neocartilage and neoperichondrium of elastic cartilage origin are regenerated 6 months after transplantation. Further, to determine whether the regenerated cartilage has the same viscoelasticity as normal auricular cartilage, we measured and evaluated tan delta $(\delta)$ as assessed by the dynamic mechanical analysis (DMA).

From the results obtained in this study, it would be possible to use further these multilayered chondrocytes in the treatment of a wide variety of craniofacial defect/deformity/ anomaly in a plastic reconstructive surgery.

\section{Materials and Methods}

This study was approved by the Ethics Committee of the Osaka City General Medical Center.

\section{Tissue sources}

The human auricular cartilage tissue used in the culture systems was obtained from the remnants of cartilage that were discarded during reconstructive surgery for treatment of microtia. The neocartilages that were examined in this study were the excess portions of neocartilages that formed in the abdominal wall and were then discarded during framework preparation when microtia patients were treated as previously reported. ${ }^{4}$

\section{Multilayered culture system}

Auricular cartilage remnants $\left(\sim 1 \mathrm{~cm}^{2}\right)$ were treated in $0.3 \%$ collagenase II (Worthington Biochemical). Samples were stirred for $4 \mathrm{~h}$ at $37^{\circ} \mathrm{C}$ and filtered through a cell strainer with a $100-\mu \mathrm{m}$ pore size (BD Bioscience), and then cells were isolated. The obtained cells were seeded at a density of $1 \times 10^{3}$ cells $/ \mathrm{cm}^{2}$, primary cultured, and then subcultured. At the third passage, cell counts became $\sim 1000$ times larger than the primary culture. Finally, the cells obtained by the subculture were seeded on the monolayer culture at a density of $2 \times 10^{4}$ cells $/ \mathrm{cm}^{2}$. In the multilayered culture system, cells were seeded three times on top of the previously seeded cells, as previously described. ${ }^{1-4}$ The cells were seeded repeatedly into the same flask every 7 days at a density of $2 \times 10^{4}$ cells $/ \mathrm{cm}^{2}$ for a total of three times. With our multilayered culture, cells of a density of $2 \times 10^{4}$ cells $/ \mathrm{cm}^{2}$ are seeded or inoculated to a total of $3 \times 10^{6}$ cells $/ 25 \mathrm{~mL}$ into a $150-\mathrm{cm}^{2}$ flask. Next, the same number of cells is further seeded or inoculated 1 week later into the same $150-\mathrm{cm}^{2}$ flask in the same manner for stratifying. One week is provided in between to allow the cells to expand during this period, and a large amount of chondrocytes is thereby obtained. Thirty $150-\mathrm{cm}^{2}$ flasks of the multilayered cultures are used per single transplantation in auricle reconstruction.

The schema of our multilayered culture system is shown in (Fig. 1). The medium used was a blend of F-12 medium and DME medium in an equal volume supplemented with $10 \%$ autoserum, FGF-2 $\left(10 \mathrm{ng} / \mathrm{mL}\right.$, Fibrast ${ }^{\circledR}$; Kaken Pharmaceuticals), penicillin $G$, streptomycin sulfate, and amphotericin B $(2.5 \mu \mathrm{g} / \mathrm{mL}$; Invitrogen). The optimal effective concentration of FGF-2 was examined in advance with data not shown. Based on the results, the FGF-2 was set to $10 \mathrm{ng} /$ $\mathrm{mL}$ in consideration that the minimum effective concentration should be used clinically. FGF-2 is clinically approved and used as a wound-healing agent in Japan.

\section{HA measurement assay}

To determine whether the cultured chondrocytes extracellularly secrete HA, which is an ECM component, the amount of HA in the medium was measured by the latex agglutination method. ${ }^{25}$ A latex was added to the medium, and an absorbance at $800 \mathrm{~nm}$ was measured by an automatic analyzer (Hitachi 7170S; Tokyo) to quantify the HA concentration.

\section{Transplantation}

As described in our previous clinical study, ${ }^{4}$ the multilayer, cultured chondrocytes were collected, drawn into a syringe, and transplanted by injection into a subcutaneous 


\section{Culture Method}
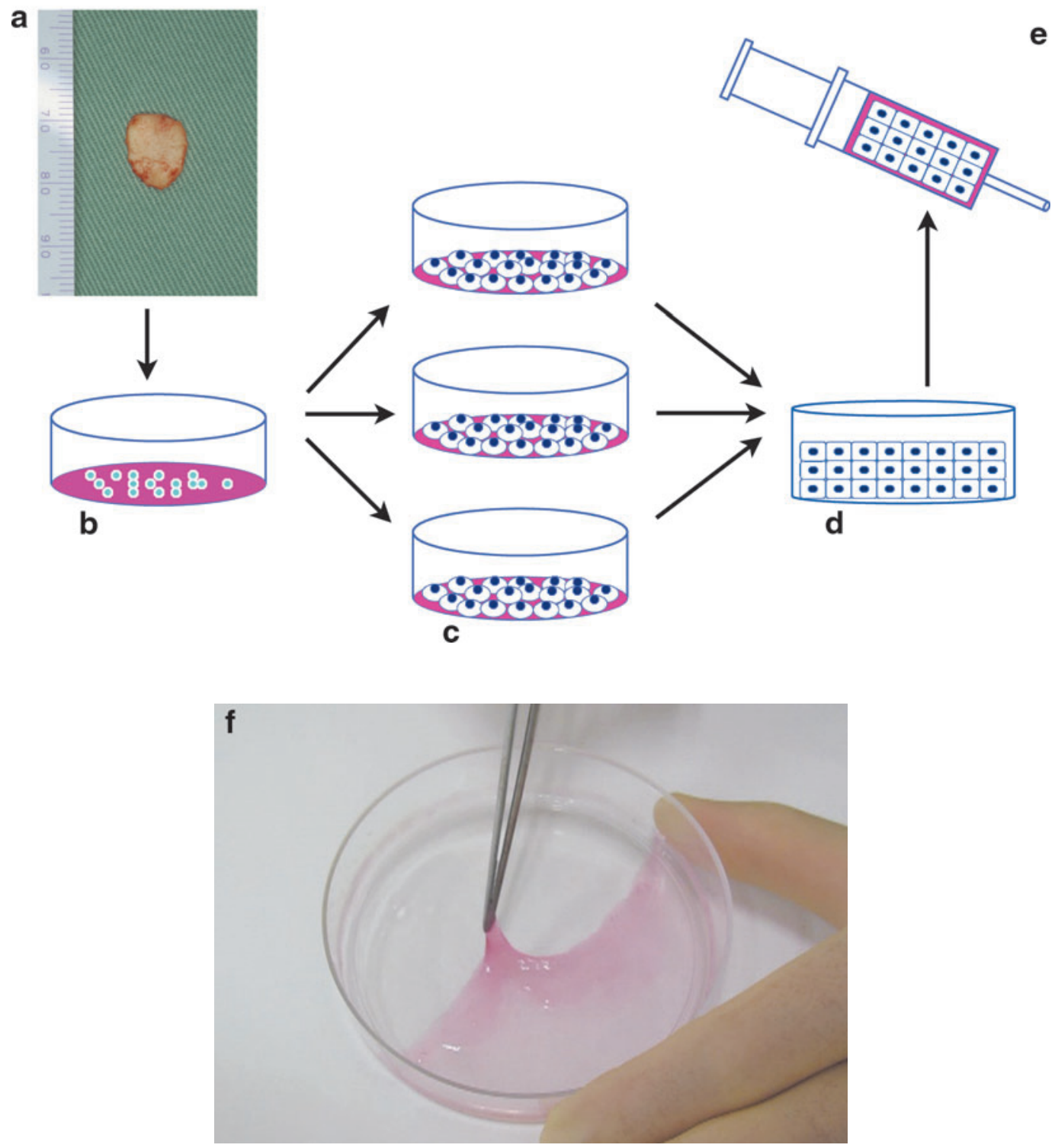

FIG. 1. A schematic of the multilayered culture systems. (a) Biopsy: elastic cartilage was harvested from auricular remnants. (b) Primary culture: the obtained chondrocytes were seeded and expanded in a flask. (c) Secondary culture: the chondrocytes were seeded at $2 \times 10^{4}$ cells $/ \mathrm{cm}^{2}$ in each flask. (d) Multilayer culture: cells were cultured three times on top of the previously seeded cells, respectively. (e) Injection: the multilayered chondrocytes were aspirated using a syringe and then injected into the patient's body for transplantation. (f) When cultured by the multilayered system, the chondrocytes form a gel-like mass that can be picked up easily in a sheet-like form from a Petri dish.

pocket on the fascia of the lower abdominal wall. It was $\sim 4$ weeks from the beginning of primary culture to transplantation. Six months later, a solid, newly regenerated cartilage block had formed subcutaneously. This neocartilage was surgically harvested, sculptured into an ear framework, and retransplanted into the microtia patient.

\section{Histological and immunohistochemical analysis}

Histological examinations were performed on the neocartilage with a neoperichondrium block that was collected 6 months after transplantation, and was fixed with 10\% neutral buffered formalin and embedded in paraffin. After deparaffinization, the specimens were stained with hematoxylin and eosin, toluidine blue, and ElasticaVan Gieson (EVG) stain. The presence of type I and II collagen was confirmed by immunohistochemistry. Human type II collagen antibodies that are cartilage specific were from Chemicon International, and Human type I collagen that presents perichondrium or are fibrous tissue specific were from Southern Biotech. For Type I and II collagen staining, an antibody dilution of 1:40 (40 $\mu \mathrm{g}$ of antibody in $100 \mu \mathrm{L})$ was used. Slides were then washed and linked to streptavidin via horseradish peroxidase (Vectastain ABC-PO) and visualized with DAB (Vectastain). Incubation without the primary antibody was used as a negative control. 


\section{Electron microscopic analysis}

Scanning electron microscopy. On the 28th culture day, the chondrocyte sheet was cut into small pieces. The specimens were fixed in a $2 \%$ glutaraldehyde/HEPES buffer $(\mathrm{pH}=7.4)$ and postfixed with a $2 \%$ OsO4/HEPES buffer. Samples were dehydrated in ethanol, cooled in liquid nitrogen, and broken apart. These specimens were replaced in isoamyl acetate, critical point dried, coated with a layer of sublimated $\mathrm{OsO}_{4}$ using an osmium plasma coater, and examined by scanning electron microscopy (SEM, JSM-6320F; JEOL).

\section{Evaluation of tan delta $(\delta)$ assessed by DMA}

DMA has been utilized to characterize auricular elastic cartilage, costal hyaline cartilage, and neocartilage viscoelastic mechanical properties. This method is nondestructive, and a specimen can be measured repeatedly at various frequencies using a dynamic mechanical analyzer when the specimen is subjected to very small nondestructive loads. ${ }^{26}$ After the thickness and width at the center of each cartilage were measured, the cartilage was placed in a DMA device (DMA 7e PerkinElmer), and then we determined viscoelastic mechanical properties (storage modulus: $G^{\prime}$ and loss modulus: $\left.G^{\prime \prime}\right)$ of the cartilage with three-point bending configuration. The parameter of $\tan \delta$ is calculated as the ratio of $\mathrm{G}^{\prime \prime} / \mathrm{G}^{\prime}$.

\section{Statistical analysis}

In statistics, a two-way analysis of variance (ANOVA) was used to compare means of two or more groups for the objective study period. The $t$-test or one-way ANOVA was also used to compare means of two or more groups at each measurement day. The Scheffe's method of multiple comparisons was used as an ad hoc analysis to evaluate the differences in all combinations of arbitrary two groups at each measurement day.

\section{Results}

\section{Proliferation and redifferentiation capability of human elastic chondrocytes in multilayered culture system}

Chondrogenic cells were obtained from elastic cartilage remnants. FGF-stimulated proliferation was consistently seen with an increase of approximately threefold. Clearly, the cell count significantly increased under the presence of FGF2 $(p<0.01)$, and it was thus demonstrated that FGF2 is involved in the proliferation of elastic chondrocytes. Next, FGF was added to a monolayer culture and a multilayer culture. Under the presence of FGF2, the concentration of $\mathrm{HA}$, which is one component of an ECM glycosaminoglycan of cartilage, clearly increased $(p<0.01)$ in comparison to the group to which FGF was not added. The HA concentration level in the medium was higher in the multilayer culture than in the monolayer culture.

The above results indicate that the addition of FGF and the multilayered culture system induced the proliferation and differentiation of elastic chondrocytes (Fig. 2).

\section{In vitro multilayered chondrocytes regenerate the $3 D$ cartilage-like structure}

Phase-contrast microscopy findings. We observed that in the monolayer culture, the chondrocytes dedifferentiate
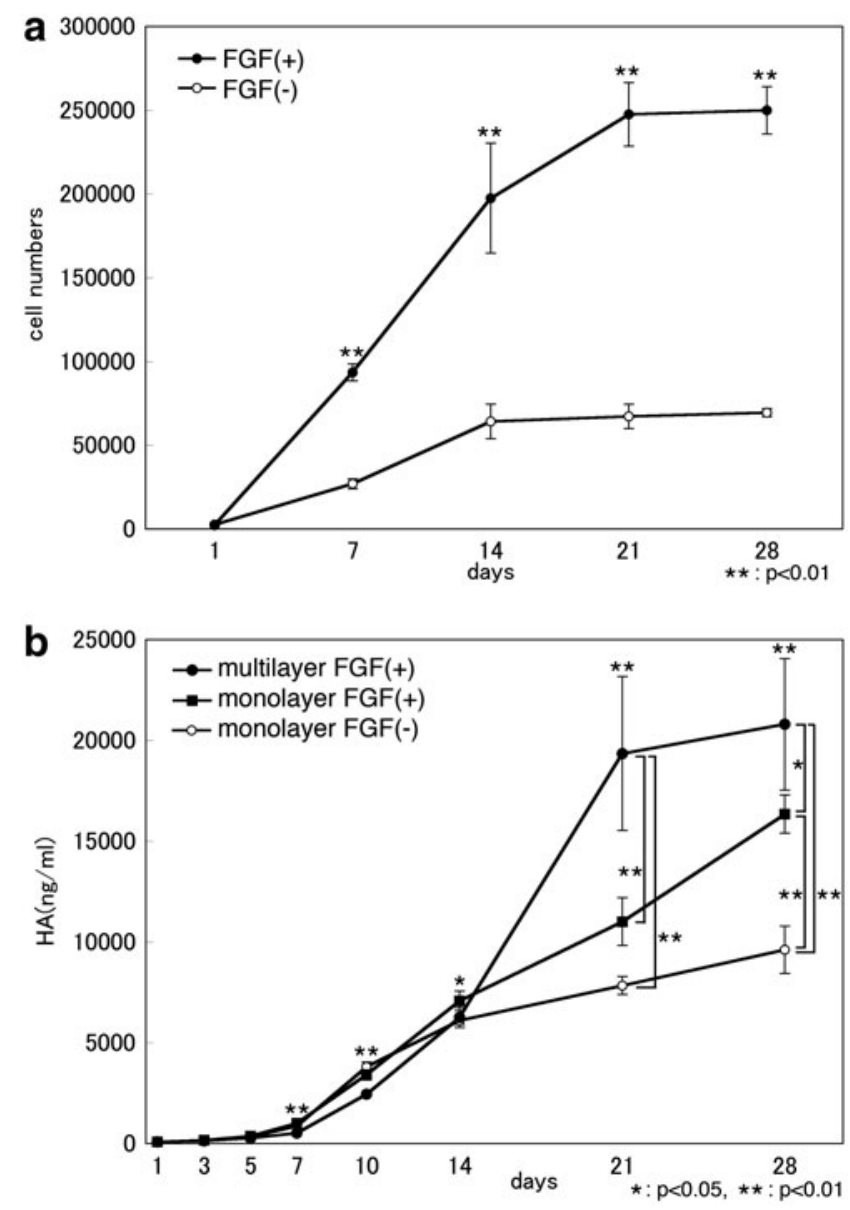

FIG. 2. Proliferation and redifferentiation capability of human elastic chondrocytes in multilayered culture system. (a) Cell proliferation of the cells in response to fibroblast growth factor-2 (FGF2) after 28 days of culture. Data are shown as the mean \pm standard deviation (SD; $N=4)$ (when compared with no addition). There were significant differences between two groups at $7,14,21$, and 28 days. That is, the cell number of cartilage of FGF $(+)$ group was significantly bigger than FGF $(-)$ group at all measurement days without the day 1. (b) Hyaluronic acid (HA) concentration level of chondrocytes in response to FGF2 in monolayer culture and multilayered culture system. Data are shown as mean $\pm S D(N=4)$ (when compared with no addition). There were significant differences among three groups on or after day 3 . At 21 days, there are significant differences between the monolayer FGF $(-)$ group and the multilayer FGF $(+)$ group, and between the monolayer FGF $(+)$ group and the multilayer FGF $(+)$ group. There was no significant difference between the monolayer FGF (-) group and the monolayer FGF (+) group. At 28 days, there were significant differences in all combinations of arbitrary two groups.

and change to flat fibroblastic cells, and the phenotype of the chondrocytes is lost. However, in the multilayered culture system, the cells overlap, connect mutually upon projecting intercellular protrusions, redifferentiate into round chondrocytes, and transform in morphology to the chondrocytelike form (Fig. 3).

Scanning electron microscopic findings. We observed that chondrocytes proliferated as a monolayer in the 
FIG. 3. Phase-contrast microscopic image and threedimensional scanning electron microscopic analysis of cells. In the conventional monolayer culture, thin and flat fibroblastlike cells adhere to the membrane as a single layer (a, c). In the multilayer culture, the chondrocytes stratified into several layers $(\mathbf{b}, \mathbf{d})$. (b) These chondrocytes maintained round phenotypes and connect to each other tightly similar to cartilagenous morphorogy. (d) These chondrocytes were thicker than the cells present in the monolayer culture. The black arrows show the focused cross section of the cells. The black triangles indicate whole cells, and the white arrows show the fibrillar components. Scale bars indicate $50 \mu \mathrm{m}$ in (a, b) and $10 \mu \mathrm{m}$ in (c, d).
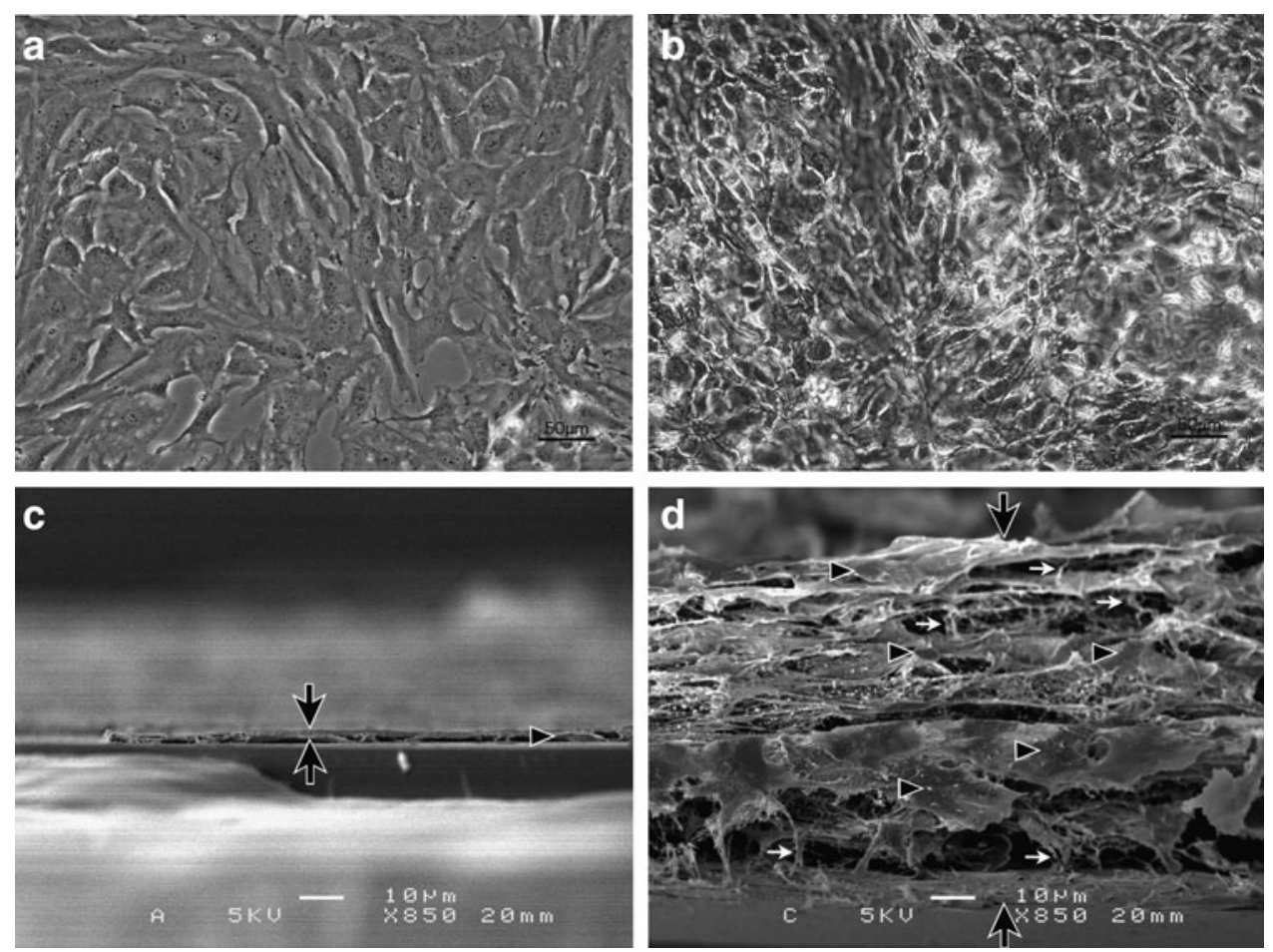

conventional culture system. In our multilayered culture system, clear stratification of the cultured chondrocyte sheets was observed (Fig. 3). In the multilayered system, the chondrocytes were connected to each other, produced ECM components and web-like networks, and were surrounded by matrix fibers and vesicles. The cytological details can be seen in the highly detailed micrographs, which show chondrocytes tightly connected to each other (Fig. 4).

\section{In vivo multilayered chondrocytes regenerate neocartilage and neoperichondrium in two-stage transplantation process}

The cultured multilayered chondrocytes regenerated a neocartilage with a neoperichondrium block in the subcutaneous pocket of a patient's abdomen post-transplantation in 6 months. The neocartilage formed in the subcutaneous region of the lower abdomen neither adhered to surrounding tissue nor dispersed to the surroundings and could be harvested readily as a single, large block. The section of the harvested neocartilage exhibited the glossy white color characteristic of cartilage tissue, and its periphery was surrounded by a neoperichondrium (Fig. 5, Supplementary Video S1 available online at www.liebertpub.com/tea).

\section{Histological analysis of the neocartilage with neoperichondrium}

With toluidine blue staining, metachromasia was depicted, and the presence of aggrecan (a cartilage-specific differentiation marker) was confirmed. Immunohistochemistry revealed that chondrocyte-derived neocartilage exhibited the human type II collagen, which shows the formation of human cartilage. Immunohistochemistry also revealed that chondrocytederived neoperichondrium exhibited the human type I colla- gen, which shows the formation of human perichondrium. EVG staining presented elastic fibers that are specific to elastic cartilage, which showed that the neocartilage block was elastic cartilage (Fig. 6). These findings together confirmed that the neocartilage block was elastic cartilage originating from the auricle cartilage, and that the cultured chondrocytes displayed normal differentiation after injection-transplantation.

\section{Mechanical evaluation of the neocartilage}

Using a DMA device, we determined the viscoelastic mechanical properties (storage modulus: $G^{\prime}$ and loss modulus: $\mathrm{G}^{\prime \prime}$ ) of the cartilage with three-point bending configuration. As a result, it was found that neocartilage $(0.2217 \pm 0.017)$ is lower in viscoelasticity than costal cartilage $(0.2557 \pm 0.0085)$, has substantially the same viscoelasticity as native auricular cartilage $(0.2157 \pm 0.0245)$, and is elastic (Fig. 7).

Neocartilage is softer than normal costal cartilage, and its viscoelasticity is equivalent to that of normal auricular cartilage.

\section{Clinical application}

There have been 12 patient cases of ear reconstruction of microtia using the cell-engineering techniques of cultured auricular chondrocytes, and in all cases, the postgrafting results are satisfactory and absorption of reconstructed neocartilage is not seen. We described one example of a longterm follow-up case in the present report.

The ear framework produced from this neocartilage block was transplanted to the auricular defect area, and an auricle with a smooth curvature and shape was subsequently configured. In the 6 years of postoperative monitoring, the neocartilage maintained a good shape without absorption (Fig. 8). 

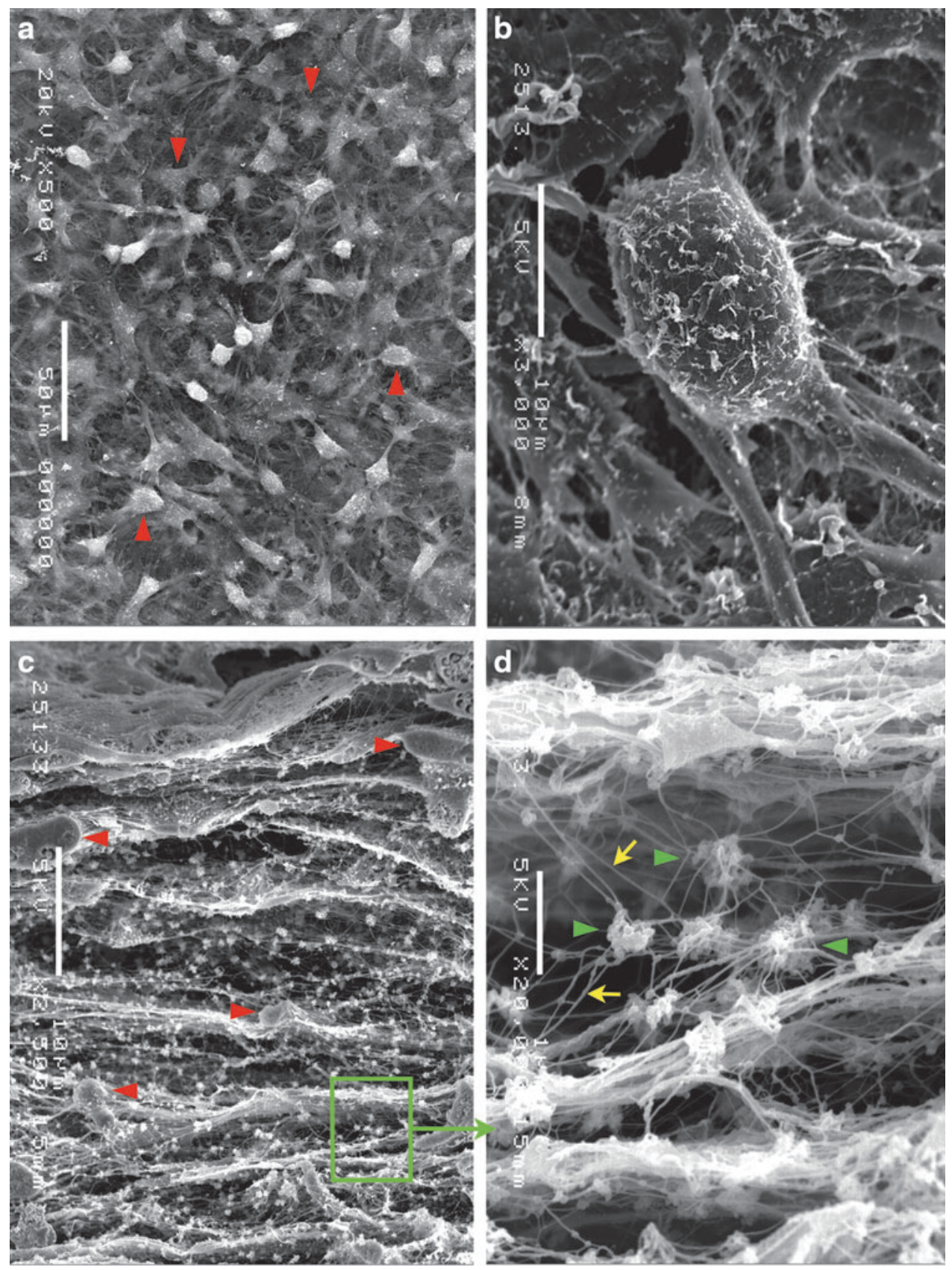

FIG. 4. Higher

magnification images from the three-dimensional scanning electron microscopic findings of multilayered chondrocytes. (a) The surface view of a chondrocyte culture shows chondrocytes (red triangles) connected to each other, creating web-like networks. (b) A higher magnification indicated a single chondrocyte with several processes extending out to the surrounding area. (c) A cross section of a chondrocyte culture shows that the chondrocytes (red triangles) overlap, and fibrillar

components create a network between the chondrocytes and many matrix vesicles. (d) A higher magnification of the area indicated by the green rectangle in (c); many matrix vesicles (green triangles) and microfibrillar components (yellow arrows) are evident. Scale bars indicate $50 \mu \mathrm{m}$ in (a), $10 \mu \mathrm{m}$ in (b, c), and $1 \mu \mathrm{m}$ in (d).

\section{Discussion}

In the human body, chondrocytes located within the matrix and mature chondrocytes gradually lose their proliferation capabilities with aging. However, when the matrix is removed with enzyme treatment, chondrocytes in culture dedifferentiate and regain their ability to proliferate. ${ }^{13-15}$ Various studies have been conducted using culture methods; however, as insufficient volumes of chondrocytes were obtained, their applications in clinical studies have been limited. To overcome this problem, the ex vivo culture of chondrocytes in combination with artificial scaffolds polylactic acid (PLA), polyglycolic acid (PGA), their copolymer (PLGA), ${ }^{27-29}$ alginate beads, agarose gel, ${ }^{30,31}$ and with biomaterial scaffolds (collagen, chitosan, fibrin, and $\mathrm{HA}^{32-38}$ ) has been investigated, but these studies did not result in any clinical applications.
Why have clinical applications been unsuccessful? Thus, instead of using artificial scaffolds, we performed cell engineering so that the cells themselves form a natural scaffold; that is, cell proliferation was promoted by addition of FGF2, and the cells were thereafter cultured by the multilayered culture system. In the results that we obtained, the group to which FGF2 was added to the medium had a clearly higher cell count than the control and also had a clearly higher level of HA. HA binds with aggrecan, a major cartilage matrix component. These results demonstrate that FGF2 causes elastic chondrocytes to proliferate and increases the production of HA, which is an ECM component of cartilage.

Also, it has been reported that although FGF-2 exhibits effects of cell proliferation and cell expansion, it suppresses cartilage phenotype expression. ${ }^{20}$ Thus, to maintain the phenotype of the proliferated chondrocytes, we arranged the 

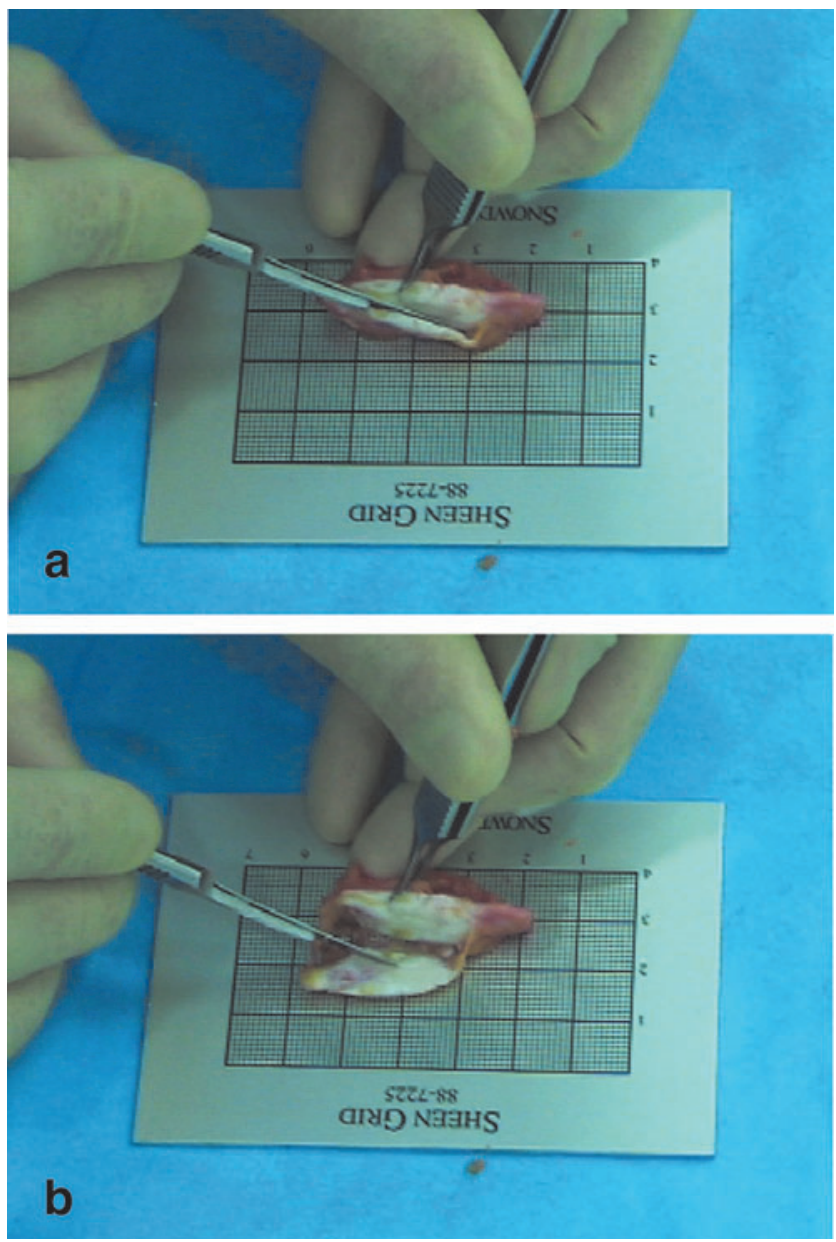

FIG. 5. In vivo multilayered chondrocytes regenerate neocartilage and neoperichondrium in a two-stage transplantation process. (a) At 6 months after transplantation, a large neocartilage with neoperichondrium was reconstructed in the patient's lower abdomen. (b) In the section of the extracted sample, the glossy neocartilage was white in color, and its periphery was surrounded by the neoperichondrium. For video presentation, see Supplementary Video S1. cells three-dimensionally in multilayers. By in vitro preparation of a state that is as close as possible to cartilage tissue, redifferentiation of the chondrocytes was induced; that is, it was found that when the chondrocytes are arranged in multilayers, the chondrocytes themselves produce ECM, and the chondrocytes connect to each other to form cartilage tissue, although immature, while forming a natural scaffold in vitro.

These results indicate that in the multilayered culture system supplemented with FGF2, the chondrocytes produce $\mathrm{HA}$ and form an ECM, and the redifferentiation of chondrocytes is induced.

As a reflection of these results, the above-described evidence was also obtained by a histological analysis. In vitro phase-difference microscopy showed that chondrocytes project protrusions and connect to each other and change from fibroblastic cells to round, chondrocyte-like forms.

In an electron microscopic analysis, SEM showed the differences in the 3D structure of cells cultured by various means. Cells cultured in a conventional monolayer formed a single layer, with no matrix components observed. Cells cultured in the multilayer system proliferated over several layers, having a rich intercellular matrix, forming networks, and producing matrix fibers and vesicles. This showed that the multilayered culture system induces cartilage tissue-like structures.

We succeeded in inducing multilayered chondrocytes before transplantation, in which the chondrocytes themselves produced a matrix structure and generated cellular networks between each other. The matrix networks developed into a natural scaffold and produced immature cartilage-like tissues in vitro.

In the in vivo histological analysis, aggrecan, a marker of cartilage differentiation, was confirmed to be present in posttransplantation tissue by toluidine blue staining, and the expression of human type II collagen, which is characteristic of cartilage matrix, was confirmed by immunostaining. By EVG staining, the presence of elastic fibers, which is a characteristic of auricular elastic cartilage, was confirmed. It was thus demonstrated that the cultured chondrocytes regenerated the origin-derived auricular cartilage after transplantation. It was also demonstrated that with the cultured chondrocytes, normal differentiation is induced and mature

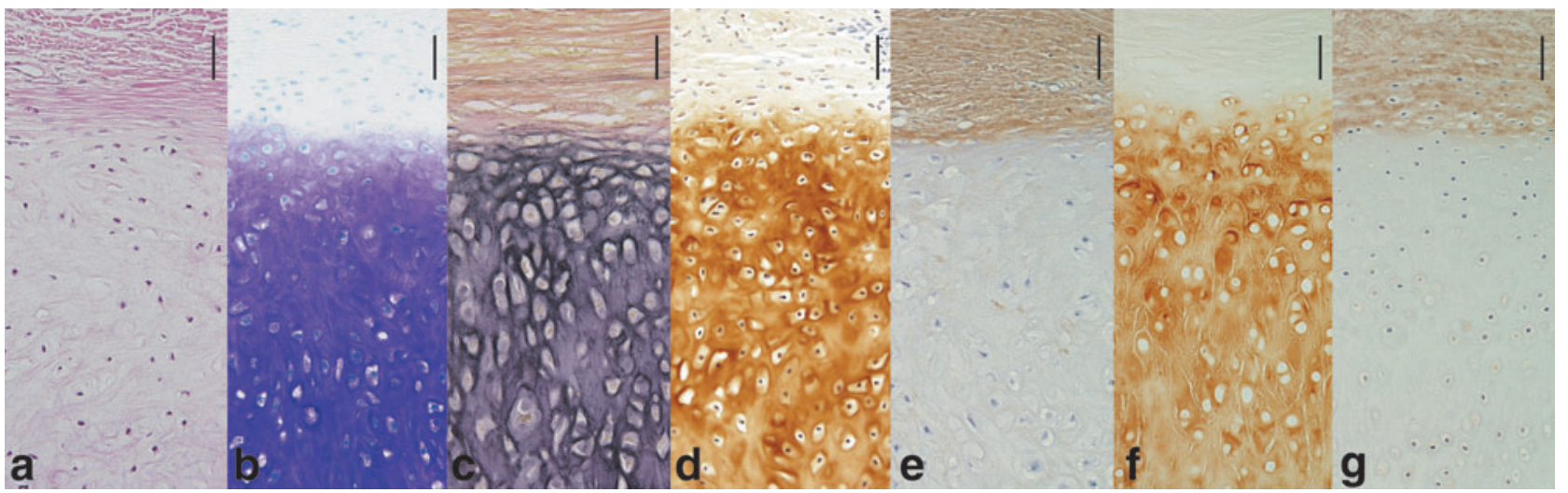

FIG. 6. Histological analysis of the neocartilage with neoperichondrium 6 months after transplantation. (a) Hematoxylin and eosin and (b) toluidine blue staining shows that neocartilage produced a large amount of aggrecan. (c) Elastica Van Gieson staining showed that neocartilage contained elastic fibers. Immunohistochemistry revealed that elastic chondrocytes regenerated neocartilage-containing type II collagen (d) and neoperichondrium-containing type I collagen (e). (f, g) Normal human auricular elastic cartilage-containing type I or type II collagen, positive control. Vessels were seen in both normal perichondrium and neoperichondrium. Also, the type I collagen was seen rich in the neocartilage matrix than in the auricular cartilage matrix. 


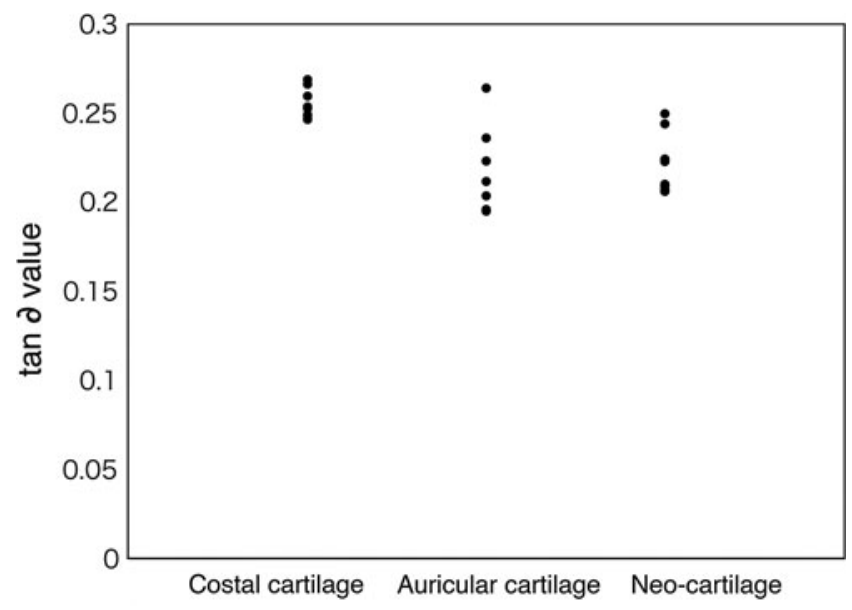

FIG. 7. Dynamic viscoelasticity measurement of neocartilage by applying three-point bending, 6 months after transplantation. $Y$-axis shows tan $\partial$ value. After transplantation, neocartilage is softer than normal costal hyaline cartilage, and its viscoelasticity is equivalent to that of a normal auricular elastic cartilage $(N=8)$. cartilage tissue is formed after transplantation. Also, by immunostaining, human type I collagen, which is stained specifically in the perichondrium or fibrous tissue, was expressed in the neoperichondrium in the same manner as in the perichondrium of normal auricular cartilage. It was thus proven that the chondrocytes themselves form perichondrium-like tissue after transplantation.

Further, as a result of performing a viscoelasticity test on the neocartilage formed after transplantation, the neocartilage was found to be softer than costal cartilage and to exhibit substantially the same viscoelasticity as auricular cartilage tissue. The mechanical characteristics of elastic cartilage were thus reproduced.

In our unique method, multilayered chondrocytes were cultured and expanded in a large volume in vitro and then transplanted into a patient's body in a two-stage transplantation process. The transplanted chondrocytes regenerate the ECM by themselves and develop into neocartilage with neoperichondrium in vivo. This finding from our study is crucial because of its likely clinical application. In future studies, we plan to investigate the applicability of this culture method with other cell types.
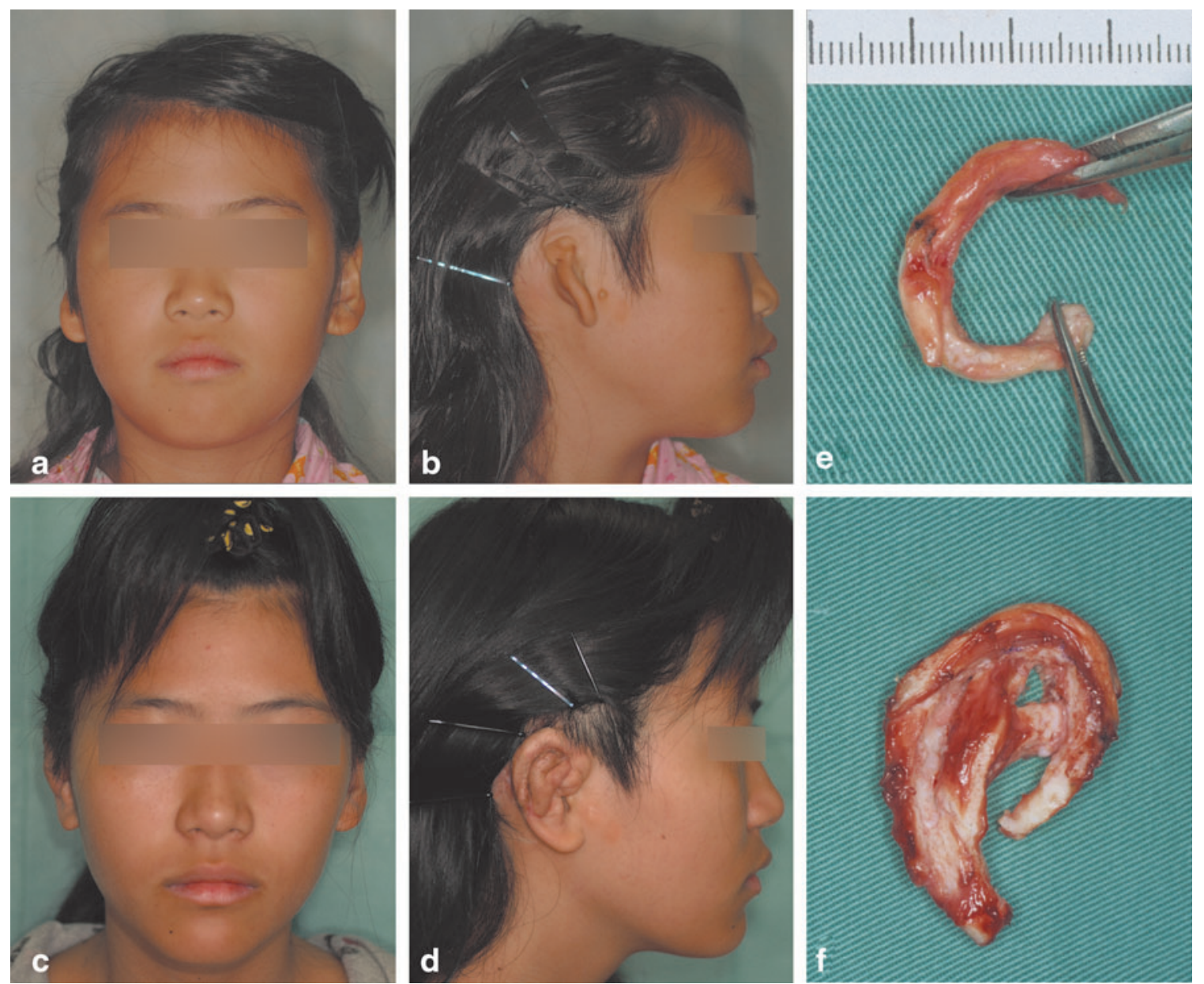

FIG. 8. Clinical results of a long-term follow-up case of microtia in a 9-year-old girl (a, b). Preoperative view (c, d). The regenerated neocartilage is elastic and can be bend into a curvilinear form (e). An ear framework, sculptured from the neocartilage block (f). Six years and 6 months after transplantation to the lower abdomen, and 5 years after transplantation to the temporal area. The photo shows the final appearance after transplanting the sculpted ear neocartilage; the patient's course has been favorable, with no absorption of the regenerated cartilage. 
We have successfully applied these cell-engineering techniques of cultured auricular chondrocytes in 12 patients. Therefore, we believe that this novel treatment method offers tangible benefits and significant advantages over traditional methods, because it avoids repeated and severe surgical burden on children and ensures successful ear regeneration, ${ }^{4,39}$ one option of the microtia treatment. These techniques can have other applications in the field of reconstructive surgery as well as in the repair of other craniofacial defects.

\section{Acknowledgment}

The authors are very thankful to Dr. Howard Green for his encouragement with our work.

\section{Disclosure Statement}

The authors declare that they have no competing financial interests.

\section{References}

1. Yanaga, H., Koga, M., Imai, K., and Yanaga, K. Clinical application of biotechnically cultured autologous chondrocytes as novel graft material for nasal augmentation. Aesthetic Plast Surg 28, 212, 2004.

2. Yanaga, H., Yanaga, K., Imai, K., Koga, M., Soejima, C., and Ohmori, K. Clinical application of cultured autologous human auricular chondrocytes with autologous serum for craniofacial or nasal augmentation and repair. Plast Reconstr Surg 117, 2019, 2006.

3. Yanaga, H., Imai, K., and Yanaga, K. Generative surgery of cultured autologous auricular chondrocytes for nasal augmentation. Aesthetic Plast Surg 33, 795, 2009.

4. Yanaga, H., Imai, K., Fujimoto, T., and Yanaga, K. Generating ears from cultured autologous auricular chondrocytes by using two-stage implantation in treatment of microtia. Plast Reconstr Surg 124, 817, 2009.

5. Vacanti, C.A., Langer, R., Schloo, B., and Vacanti, J.P. Synthetic polymers seeded with chondrocytes provide a template for new cartilage formation. Plast Reconstr Surg 88, 753, 1991.

6. Cao, Y., Vacanti, J.P., Paige, K.T., Upton, J., and Vacanti, C.A. Transplantation of chondrocytes utilizing a polymercell construct to produce tissue-engineered cartilage in the shape of a human ear. Plast Reconstr Surg 100, 297, 1997.

7. Rodriguez, A., Cao, Y.L., Ibarra, C., Pap, S., Vacanti, M., Eavey, R.D., et al. Characteristics of cartilage engineered from human pediatric auricular cartilage. Plast Reconstr Surg 103, 1111, 1999.

8. Ting, V., Sims, C.D., Brecht, L.E., McCarthy, J.G., Kasabian, A.K., Connelly, P.R., et al. In vitro prefabrication of human cartilage shapes using fibrin glue and human chondrocytes. Ann Plast Surg 40, 413, 1998.

9. van Osch, G.J., van der Veen, S.W., and Verwoerd-Verhoef, H.L. In vitro redifferentiation of culture-expanded rabbit and human auricular chondrocytes for cartilage reconstruction. Plast Reconstr Surg 107, 433, 2001.

10. Tay, A.G., Farhadi, J., Suetterlin, R., Pierer, G., Heberer, M., and Martin, I. Cell yield, proliferation, and postexpansion differentiation capacity of human ear, nasal, and rib chondrocytes. Tissue Eng 10, 762, 2004.
11. Kamil, S.H., Kojima, K., Vacanti, M.P., Bonassar, L.J., Vacanti, C.A., and Eavey, R.D. In vitro tissue engineering to generate a human-sized auricle and nasal tip. Laryngoscope 113, 90, 2003.

12. Chang, S.C., Tobias, G., Roy, A.K., Vacanti, C.A., and Bonassar, L.J. Tissue engineering of autologous cartilage for craniofacial reconstruction by injection molding. Plast Reconstr Surg 112, 793, 2003.

13. von der Mark, K., Gauss, V., von der Mark, H., and Müller, P. Relationship between cell shape and type of collagen synthesised as chondrocytes lose their cartilage phenotype in culture. Nature 267, 531, 1977.

14. Schiltz, J.R., Mayne, R., and Holtzer, H. The synthesis of collagen and glycosaminoglycans by dedifferentiated chondroblasts in culture. Differentiation 1, 97, 1977.

15. Aulthouse, A.L., Beck, M., Griffey, E., Sanford, J., Arden, K., and Machado, M.A., et al. Expression of the human chondrocyte phenotype in vitro. In Vitro Cell Dev Biol 25, 659, 1989.

16. Binderman, I., and Sömjen, D. 24,25-Dihydroxycholecalciferol induces the growth of chick cartilage in vitro. Endocrinology 115, 430, 1984.

17. Giovannini, S., Diaz-Romero, J., Aigner, T., Heini, P., MainilVarlet, P., and Nesic, D. Micromass co-culture of human articular chondrocytes and human bone marrow mesenchymal stem cells to investigate stable neocatilage tissue formation in vitro. Eur Cells Mater 20, 245, 2010.

18. Roy, R., Kudryashov, V., Doty, S.B., Binderman, I., and Boskey, A.L Differentiation and mineralization of murine mesenchymal C3H10T1/2 cells in micromass culture. Differentiation 79, 211, 2010.

19. Arévalo-Silva, C.A., Cao, Y., Weng, Y., Vacanti, M., Rodríguez, A., Vacanti, C.A., et al. The effect of fibroblast growth factor and transforming growth factor-beta on porcine chondrocytes and tissue-engineered autologous elastic cartilage. Tissue Eng 7, 81, 2001.

20. Weksler, N.B., Lunstrum, G.P., Reid, E.S., and Horton, W.A. Differential effects of fibroblast growth factor (FGF) 9 and FGF2 on proliferation, differentiation and terminal differentiation of chondrocytic cells in vitro. Biochem J 342, 677, 1999.

21. Jakob, M., Démarteau, O., Schäfer, D., Hintermann, B., Dick, W., Heberer, M., et al. Specific growth factors during the expansion and redifferentiation of adult human articular chondrocytes enhance chondrogenesis and cartilaginous tissue formation in vitro. J Cell Biochem 81, 368, 2001.

22. Praul, C.A., Ford, B.C., and Leach, R.M. Effect of fibroblast growth factors $1,2,4,5,6,7,8,9$, and 10 on avian chondrocyte proliferation. J cell Biochem 84, 359, 2002.

23. Mandl, E.W., van der Veen, S.W., Verhaar, J.A., and van Osch, G.J. Serum-Free medium supplemented with highconcentration FGF2 for cell expansion culture of human ear chondrocytes promotes redifferentiation capacity. Tissue Eng 8, 573, 2002.

24. Mandl, E.W., Jahr, H., Koevoet, J.L., van Leeuwen, J.P., Weinans, H., Verhaar, J.A., et al. Fibroblast growth factor-2 in serum-free medium is a potent mitogen and reduces dedifferentiation of human ear chondrocytes in monolayer culture. Matrix Biol 23, 231, 2004.

25. Engström-Laurent, A., Lööf. L., Nyberg, A., and Schröder, T. Increased serum levels of hyaluronate in liver disease. Hepatology 5, 638, 1985.

26. Meanrd, K.P. Dynamic Mechanical Analysis: A Practical Introduction. New York: CRC Press, 2008. pp. 1-15. 
27. Ma, Z., Gao, C., Gong, Y., and Shen, J. Cartilage tissue engineering PLLA scaffold with surface immobilized collagen and basic fibroblast growth factor. Biomaterials 26, 1253, 2005.

28. Freed, L.E., Marquis, J.C., Nohria, A., Emmanual, J., Mikos, A.G., and Langer, R. Neocartilage formation in vitro and in vivo using cells cultured on synthetic biodegradable polymers. J Biomed Mater Res 27, 11, 1993.

29. Lu, L., Stamatas, G.N., and Mikos, A.G. Controlled release of transforming growth factor beta1 from biodegradable polymer microparticles. J Biomed Mater Res 5, 440, 2000.

30. Liu, H., Lee, Y.W., and Dean, M.F. Re-expression of differentiated proteoglycan phenotype by dedifferentiated human chondrocytes during culture in alginate beads. Biochimi Biophys Acta 1425, 505, 1998.

31. Benya, P.D., and Shaffer, J.D. Dedifferentiated chondrocytes reexpress the differentiated collagen phenotype when cultured in agarose gels. Cell 30, 215, 1982.

32. Chang, C.H., Liu, H.C., Lin, C.C., Chou, C.H., and Lin, F.H. Gelatin-chondroitin-hyaluronan tri-copolymer scaffold for cartilage tissue engineering. Biomaterials 24, 4853, 2003.

33. Ma, L., Gao, C., Mao, Z., Zhou, J., and Shen, J. Enhanced biological stability of collagen porous scaffolds by using amino acids as novel cross-linking bridges. Biomaterials 25, 2997, 2004.

34. van Susante, J.L.C., Pieper, J., Buma, P., van Kuppevelt, T.H., van Beuningen, H., van Der Kraan, P.M., et al. Linkage of chondroitin-sulfate to type I collagen scaffolds stimulates the bioactivity of seeded chondrocytes in vitro. Biomaterials 22, 2359, 2001.
35. Xia, W., Liu, W., Cui, L., Liu, Y., Zhong, W., Liu, D., et al. Tissue engineering of cartilage with the use of chitosangelatin complex scaffolds. J Biomed Mater Res B Appl Biomater 71, 373-80, 2004.

36. Suh, J.K., and Matthew, H.W. Application of chitosan-based polysaccharide biomaterials in cartilage tissue engineering: a review. Biomaterials 21, 2589, 2000.

37. Perka, C., Arnold, U., Spitzer, R.S., and Lindenhayn, K. The use of fibrin beads for tissue engineering and subsequential transplantation. Tissue Eng 7, 359, 2001.

38. Lisignoli, G., Cristino, S., Piacentini, A., Toneguzzi, S., Grassi, F., Cavallo, C., et al. Cellular and molecular events during chondrogenesis of human mesenchymal stromal cells grown in a three-dimensional hyaluronan based scaffold. Biomaterials 26, 5677, 2005.

39. Green, H. Therapy with Cultured Cells. Singapore: Pan Stanford Publishing Pte. Ltd. www.panstanford.com, 2010, pp. 55-56.

Address correspondence to: Hiroko Yanaga, M.D., Ph.D. Yanaga Clinic and Tissue Culture Laboratory 1-2-12 Tenjin, Chuo-ku Fukuoka 810-0001 Japan

E-mail: force@yanaga-cl.com

Received: June 27, 2011

Accepted: May 2, 2012

Online Publication Date: July 3, 2012 